\title{
Convolution and Filtering in Fractional Fourier Domains
}

\author{
Haldun M. OzaKTas, ${ }^{1}$ Billur BaRSHAN ${ }^{1}$ and David MENDlOVIC ${ }^{2}$ \\ ${ }^{1}$ Electrical Engineering Department, Bilkent University, 06533 Bilkent, Ankara, Turkey \\ ${ }^{2}$ Faculty of Engineering, Tel-Aviv University, 69978 Tel-Aviv, Israel \\ (Accepted July 1, 1994)
}

Fractional Fourier transforms, which are related to chirp and wavelet transforms, lead to the notion of fractional Fourier domains. The concept of filtering of signals in fractional domains is developed, revealing that under certain conditions one can improve upon the special cases of these operations in the conventional space and frequency domains. Because of the ease of performing the fractional Fourier transform optically, these operations are relevant for optical information processing.

Key words : optical information processing, Fourier optics, fractional Fourier transforms, spatial filtering

\section{Introduction}

Whenever we are confronted with an operator, it is natural to inquire into the effect of repeated applications of that operator, which might be considered as its integer powers. A further extension is to inquire what meaning may be attached to fractional powers of that opertor. The fractional Fourier transform was defined mathematically by McBride and Kerr. ${ }^{1)}$ In Refs. 2-6), it is shown how the two-dimensional fractional Fourier transform can be realized optically and various mathematical and physical properties are discussed.

The definition of the ath order fractional Fourier transform $\widetilde{F}^{a}[f]$ can be cast in the form of a general linear transformation with kernel $B_{a}\left(x, x^{\prime}\right)$ :

$$
\begin{aligned}
& \qquad \begin{array}{l}
\left(\mathfrak{F}^{a}[f(x)]\right)(x)=\int_{-\infty}^{\infty} B_{a}\left(x, x^{\prime}\right) f\left(x^{\prime}\right) \mathrm{d} x^{\prime}, \\
B_{a}\left(x, x^{\prime}\right)=\frac{\mathrm{e}^{\mathrm{i}(\pi \hat{\phi} / 4-\phi / 2)}}{|\sin \phi|^{1 / 2}} \\
\quad \times \exp \left[\mathrm{i} \pi\left(x^{2} \cot \phi-2 x x^{\prime} \csc \phi+x^{\prime 2} \cot \phi\right)\right],
\end{array} \\
& \text { for } 0<|\phi|<\pi(\text { i.e. } 0<|a|<2), \text { where } \\
& \phi=a \pi / 2
\end{aligned}
$$

and

$$
\hat{\phi}=\operatorname{sgn}(\sin \phi) \text {. }
$$

The kernel is defined separately for $a=0$ and $a=2$ as $B_{0}\left(x, x^{\prime}\right)=\delta\left(x-x^{\prime}\right)$ and $B_{2}\left(x, x^{\prime}\right)=\delta\left(x+x^{\prime}\right)$ respectively.

The kernel $B_{a}\left(x, x^{\prime}\right)$ is a chirp function, allowing the above transformation to be interpreted as a coordinate transformation in which the chirp functions play the role of basis functions. Based on this concept, a formulation of fractional Fourier transform can be characterized by the following properties:

1. Basis functions in the ath domain, be they delta functions or harmonics, are in general chirp frunctions in any other $\left(a^{\prime}\right)$ th domain.

2. The representation of a signal in the $a$ th domain can be obtained from the representation in the $\left(a^{\prime}\right)$ th domain by taking the inner product (projection) of the representation in the $\left(a^{\prime}\right)$ th domain with basis functions in the target ath domain.

3. This operation, having the form of a chirp transform, is equivalent to taking the $\left(a-a^{\prime}\right)$ th fractional Fourier transform of the representation in the $\left(a^{\prime}\right)$ th domain.

The relationship of fractional Fourier transform to chirp transforms provides the basis of the concept of fractional domains, which are generalizations of the conventional space and frequency domains. The relationship to wavelet transoforms is discussed in Ref. 6).

\section{Filtering in Fractional Domains}

Now we move on to discussing filtering in fractional domains. We will see that under certain circumstances, noise separation can be realized effectively in fractional Fourier domains: Fractional Fourier transform can be used to separate signals which cannot be separated in ordinary coordinate and frequency domains. For instance, consider the signal and noise components shown in Fig. 1. Their projections on both coordinate and frequency axes overlap, however, their projections on the axis corresponding to the ath fractional Fourier domain do not. Thus, the signal can be separated from the noise easily.

Now let us give some more concrete examples. Consider the signal

distorted additively by

$$
\exp \left[-\pi(x-4)^{2}\right]
$$

$$
\exp \left(-\mathrm{i} \pi x^{2}\right) \operatorname{rect}(x / 16) \text {. }
$$

The magnitude of their sum is displayed in part a of Fig. 2. These signals overlap in the frequency domain as well. In part $b$, we show their $a=0.5$ th fractional Fourier transform. We observe that the signals are separated in this domain. The chirp distortion is transformed into a peaked function which does not exhibit significant overlap with the signal transform, so that it can be blocked out by a simple mask (part c). Inverse transforming to the original domain, we obtain the desired signal nearly perfectly cleansed of the chirp distortion (part $\mathrm{d}$ ).

Now we consider a slightly more involved example in which the distorting signal is also real. The signal

is distorted additively by

$$
\exp \left(-\pi x^{2}\right)
$$

$$
\cos \left[2 \pi\left(x^{2} / 2-4 x\right)\right] \operatorname{rect}(x / 8),
$$

as shown in part a of Fig. 3. The $a=0.5$ th transform is shown in part $b$. One of the complex exponential chirp components of the cosine chirp has been separated in this 


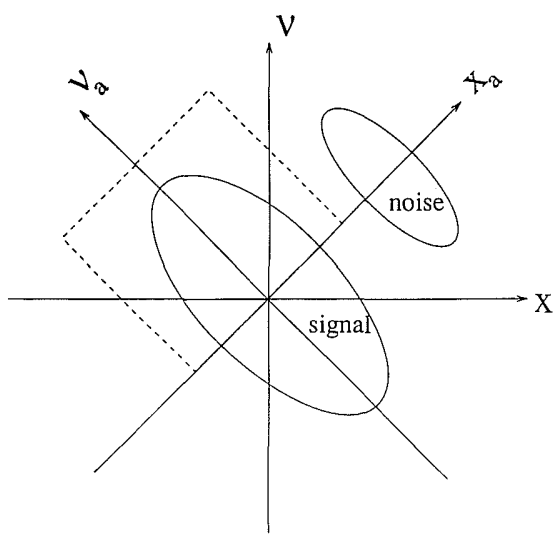

Fig. 1.
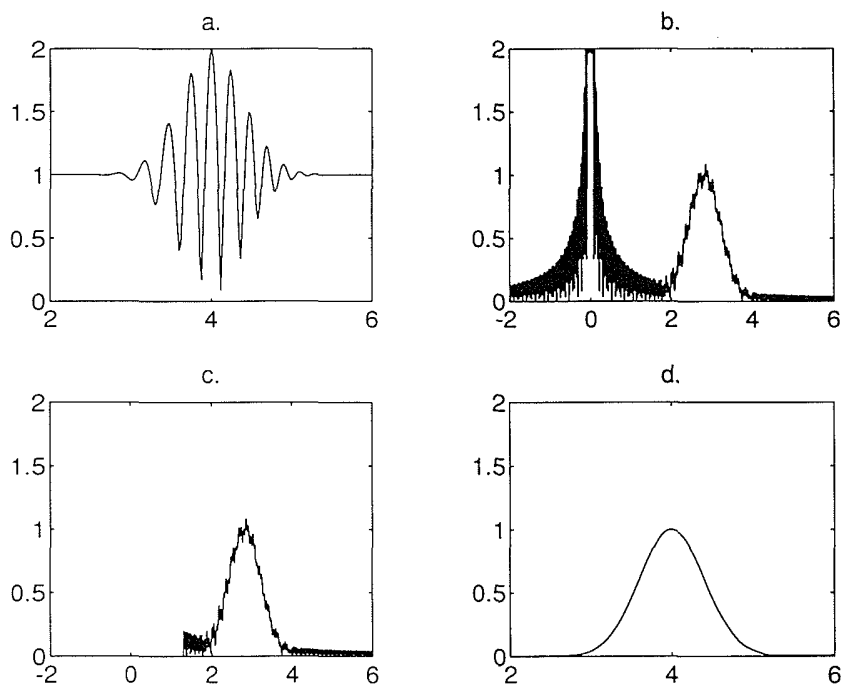

Fig. 2.

domain and can be masked away, but the other still distorts the transform of the Gaussian. After masking out the separated chirp component (not shown), we take that $a=-1$ st transform (which is just an inverse Fourier transform), to arrive at the $a=-0.5$ th domain (part c). Here the other chirp component is separated and can be blocked out by another simple mask. Finally, we take the 0.5 th transform to come back to our home domain (part d), where we have recovered our Gaussian signal, with a small error.

The examples above have been limited to chirp distortions which are particularly easy to separate in a fractional Fourier domain (just as pure harmonic distortion is particularly easy to separate in the ordinary Fourier domain). However, it is possible to filter out more general types of distortion as well. In some cases this may require several consecutive filtering operations in several fractional domains of different order. ${ }^{6}$ ) There is nothing special about our choice of Gaussian signals other than the fact that they allow easy analytical manipulation. Also, there is nothing special about the 0.5 th domain. It just turns out that this is the domain of choice for the examples considered above.

In the above examples we have demonstrated that the method works, but did not discuss what led us to transform to a particular domain and what gave us the
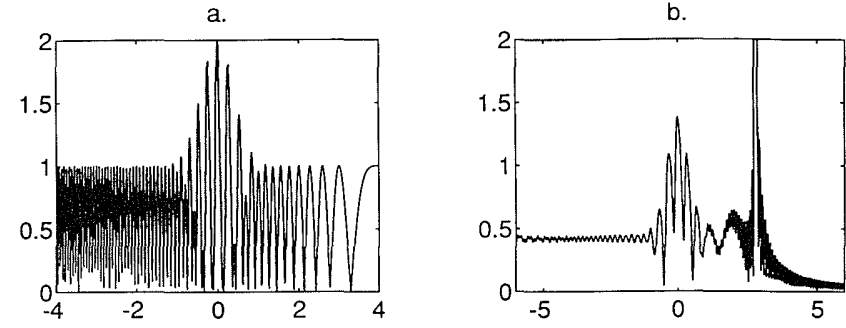

c.

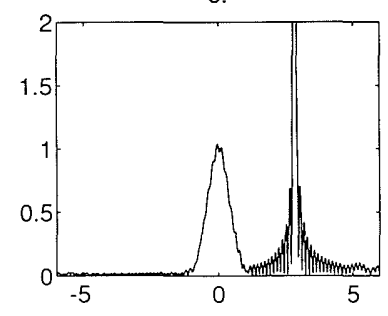

d.

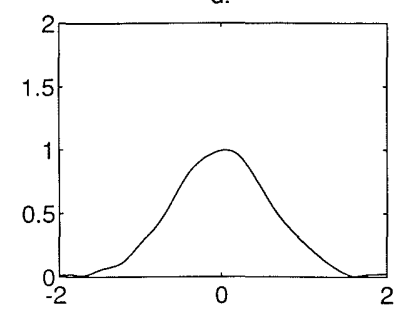

Fig. 3.

confidence that doing so will get rid of the distortion. This becomes very transparent once one understands the relationship between the fractional Fourier transform and the Wigner distribution. This relationship, as well as the general philosophy behind such filtering operations is disucced in Ref. 6).

\section{Conclusions}

The concept of fractional Fourier transform is related to chirp and wavelet transforms, as well as being intimately connected to the concept of space-frequency distributions. This leads to the notion of fractional Fourier domains, which are discussed at length in Ref. 6). In this papar, we have shown numerical examples in which filtering in a fractional domain can enable effective noise elimination. Because of the ease of performing the fractional Fourier transform optically, these operations are relevant for optical information processing.

The concept of multiplexing in fractional domains is also investigated in Ref. 6), showing that for certain signal Wigner distributions, efficient multiplexing can be realized in fractional domains.

In most of this paper, we work with continuous signals which are represented as functions of space or spatial frequency. Temporal interpretations of our discussions can be provided easily by those interested in them.

\section{Acknowledgments}

We acknowledge the support of NATO under the Science for Stability Program.

\section{References}

1) A.C. McBride and F.H. Kerr: IMA J. Appl. Math. 39 (1987) 159.

2) H.M. Ozaktas and D. Mendlovic: Opt. Commun. 101 (1993) 163.

3) D. Mendlovic and H.M. Ozaktas: J. Opt. Soc. Am. A 10 (1993) 1875.

4) H.M. Ozaktas and D. Mendlovic: to appear in J. Opt. Soc. Am. A.

5) A.W. Lohmann: J. Opt. Soc. Am. A 10 (1993) 2181.

6) H.M. Ozaktas, B. Barshan, D. Mendlovic and L. Onural: to appear in J. Opt. Soc. Am. A. 